\title{
Formation of Coffee Stains on Porous
}

\section{Surfaces}

\author{
Rui Dou and Brian Derby*
}

School of Materials, University of Manchester, Oxford Rd., Manchester, M13 9PL, UK.

brian.derby@manchester.ac.uk

\section{ABSTRACT}

During the drying of drops of nanoparticle suspensions, segregation can occur by internal fluid flows towards the contact line, if the contact line is pinned. This leads to a characteristic ring deposit or coffee stain. On solid substrates coffee staining can be eliminated through the use of solvent mixtures that promote Marangoni flows to oppose these drying induced flows. Here it is shown that a suspension, optimized to eliminate the formation of coffee stains on a range of solid surfaces, shows coffee staining on a number of porous surfaces. This behavior is shown to be consistent with a mechanism of fluid removal through capillary flow (draining) of the solvent into the porous substrate, combined with filtration of the particles by the small pore size, in addition to the flow from solvent evaporation. The extent of capillary driven coffee staining is a function of substrate pore size: if the pore size is small, capillary 
flow is slow, reducing the observed coffee staining. However, if the pore size is too large, the nanoparticles are absorbed into the material along with the draining solute and no deposition of particles is observed.

\section{INTRODUCTION}

A coffee stain (or coffee ring) occurs if the contact line at the edge of a drying fluid drop is pinned, preventing the drop from retracting when its volume is reduced by loss of solvent. ${ }^{1}$ Contact line pinning occurs because heterogeneities in surface energy or surface profile (roughness) result in a hysteresis between the advancing and receding contact angle. ${ }^{2,3}$ This leads to a static contact line during the initial stages of drying; because the drop cannot retract, there is a compensating flow of material from the drop centre to the edges, leading to

a replenishment of liquid and enhanced deposition/precipitation close to the pinned edge. ${ }^{1,4}$ Under appropriate conditions, the outward radial flow can lead to almost complete depletion at the drop centre with the resulting deposit in the form of a ring at the initial contact line. However, despite coffee staining being a result of contact line pinning, Davis showed that pinning is beneficial if lines are formed from linear patterns of overlapping drops; in which case contact line pinning stabilizes the resulting liquid bead against capillary driven break up into discrete sessile droplets.

To date, all published studies on the phenomenon of coffee staining have considered the behavior of drops of solutions, or particle dispersions, drying on relatively smooth, flat, 
impermeable solid surfaces. Although such a configuration is of importance in a number of technologies, the motivation for this work is to understand the behavior of small drops of liquid, containing solute or particles in suspension, drying on a porous or permeable surface. Such a drop/substrate configuration is possible in a number of future potential commercial applications for inkjet printing such as:

- The direct manufacture of 3-D ceramic structures from overprinting sequential layers of powder suspensions onto previously printed and dried deposits. ${ }^{7}$

- The printing of electronic circuits on disposable paper electrodes for low cost applications such as RFID tags. ${ }^{8}$

- The manufacture of electronic circuits on textile substrates for applications such as electronically functional clothing. ${ }^{9}$

In all these cases the formation of a coffee stain could be influenced by the porosity of the substrate. This is because there is an additional fluid removal mechanism that occurs through capillary infiltration into the pores, draining the drop. It has previously been shown that liquid drops, printed onto porous substrates, spread to their full extent over timescales much shorter than that for infiltration into fine powder beds. ${ }^{10,11}$ Thus the drying of a sessile drop on a porous surface occurs through two different mechanisms of solvent removal, evaporation or drainage. This study investigates whether draining of a fluid into a powder bed changes its behavior with regard to coffee staining. 
Deegan proposed a simple model to explain this phenomenon, ${ }^{1,4}$ which considers how the height $h$, at a given radial position $r$, is influenced by the local evaporation flux $J(r, t)$, as a function of time $t$, and the radial flow velocity required to maintain the pinned contact line $v$ :

$$
\rho \frac{d h}{d t}=-\rho \frac{1}{r} \frac{\partial}{\partial r}(r h v)-J(r, t)\left(1+\left(\frac{\partial h}{\partial r}\right)^{2}\right)^{1 / 2}
$$

To solve this, Deegan assumed the drop to be sufficiently small that its shape could be approximated to a spherical cap of fixed base radius $R$, and derived a quasi-static solution to the evaporation flux with:

$J(r) \propto(R-r)^{-\lambda}$

with $\lambda=\frac{\pi-2 \theta}{2 \pi-\theta}$

where $\theta$ is the contact angle. Equation 2 is obtained by considering the influence of the large dry area surrounding the droplet, which enhances evaporation from the drop edges. ${ }^{1}$ However later experimental studies found that coffee staining also occurred when conditions resulted in uniform evaporation across the surface of the drop and was only suppressed when evaporation was confined to the centre of the drying drop. ${ }^{4}$

Deegan's analysis of the fluid motion during the drying of a sessile drop made a large number of simplifying assumptions. The most important was to consider a uniform average radial flow with no variation in the vertical direction. However, during evaporation, there are 
also changes in temperature driven by the phase change, this can lead to local gradients in temperature and a second fluid flow driven by Marangoni convection (i.e. gradients in surface tension induced by gradients in temperature). Fischer developed a more sophisticated treatment of evaporation than was considered by Deegan and also included flow during drying that was generated by Marangoni convection. ${ }^{12}$ He also noted that Deegan's solution (equation 2) implies a singularity in the evaporation flux at the contact line and argued that a more physically correct model would have a zero evaporation flux here, where the height of the liquid is zero. Fischer modeled flow within the drying drop using lubrication theory and numerical simulations were carried out to study the influence of different models of the radial dependence of the evaporation flux. These showed that if the evaporation flux is constant over the drop surface, or if there was an enhancement of flux towards the edge, there will be a net outward radial flow of fluid; only if the evaporation is confined to the centre of the drop is the radial flow suppressed during drying. Later work by $\mathrm{Hu}$ and Larson modeled the temperature distribution in a drying drop and found results that were consistent with Fischer's model. ${ }^{13}$ Ozawa et al considered radial flow during drying drops of highly concentrated solutions/suspensions but in the absence of Marangoni flows. ${ }^{14}$ They proposed that segregation was enhanced by the gelation of the suspension close to the contact line, which occurs because of increased solute concentration from an outward radial flow. Thus, coffee staining is expected to be more pronounced as the concentration of solute/suspendant increases or when the evaporative flux is increased (e.g. through an increase in temperature).

$\mathrm{Hu}$ and Larson, however, noted that Marangoni convection is extremely sensitive to small levels of surfactant impurity and predicted that surface concentrations as small as 300 
molecules $/ \mu \mathrm{m}^{2}$ could completely suppress Marangoni flow. ${ }^{13}$ This prediction was supported by an experimental study of droplet drying that found complete suppression of any coffee stains by Marangoni flow when an ultra-pure organic solvent was used. ${ }^{15}$ These results were used by $\mathrm{Hu}$ and Larson to propose that the common reports of coffee staining during the drying of drops, especially when aqueous solvents are used, is because the solvents are rarely pure and that contamination reduces Marangoni convection, hence allowing the radial flow that promotes segregation to the contact line. However, this assertion of the influence of impurities on drying induced flows in aqueous systems is a matter of dispute in the literature as reviewed by Poulard et al. ${ }^{16}$

Ozawa et al suggested that reducing the evaporation rate will result in a reduction in segregation ${ }^{14}$ and this approach was used by Soltman and Subramanian who found that reducing the substrate temperature (and hence evaporation rate) resulted in a reduction in segregation and an elimination of coffee stains. ${ }^{17}$ Earlier work found that segregation during the drying of $\mathrm{ZrO}_{2}$ suspensions was increased with increasing substrate temperature and hence increased evaporation rate, ${ }^{18}$ consistent with the model of Ozawa. However, Wang et al found the opposite trend during the inkjet printing of carbon nanotubes (CNT), with segregation and coffee staining reduced when the substrate temperature was increased. ${ }^{19}$ The difference between the thermal behavior of CNT suspensions and those made from uniform particles may be due to their shape; recent work considering the drying of drops of particles in suspension, has found that surface flows are impeded with increasing particle aspect ratio. ${ }^{20}$ An alternative mechanism for the suppression of coffee stains was proposed by Shen et al. ${ }^{21}$ They proposed that coffee stains form if the solvent is removed over a time scale that 
allows sufficient time for the particles in suspension to rearrange and deposit near the contact line. In which case there is a critical droplet size below which solvent removal occurs sufficiently rapidly to suppress segregation and this would be expected to become more likely as the substrate temperature is increased.

The motivation for this work is to better understand the phenomenon of coffee staining that may occur after the printing of particulate suspensions for functional applications on a range of possible substrate materials. Inks developed for this purpose are likely to be complex formulations containing viscosity modifiers, dispersants/surfactants, humectants, in addition to functional materials or particles in solution or suspension. The behavior of such complex mixtures is not readily predicted by modeling and a more empirical approach has been used to develop inks that suppress segregation during drying after inkjet printing. ${ }^{22}$ Practical methods to reduce coffee staining during the drying of inkjet printed solutions or dispersions use mixtures of solvents, with significantly different vapor pressures. On drying, the different relative evaporation rates of the solvent's components across a drop will induce concentration gradients in the fluid, these lead to Marangoni flows from induced surface tension gradients. $^{23-25}$

Therefore, it is clear that although the fundamental underlying mechanisms that generate the observed radial flow during drying is well understood, at least for the case when drops dry on a solid surface, there are still considerable uncertainties in the precise flow patterns that occur during the drying of a given formulation of solution or suspension. In many applications of inkjet printing the substrate is porous, e.g. paper, which is the ubiquitous substrate for 
graphics output, and textiles. Such porous materials are attracting increasing interest as potential substrates for low cost printed electronics or disposable applications such as RFID tags. ${ }^{8,9}$ In an earlier report, the authors investigated the fabrication of 3-dimensional objects by sequential inkjet printing powder suspensions onto previously dried layers. ${ }^{22}$ In this case coffee straining was suppressed on solid surfaces through the use of appropriate solvent mixtures; however, after printing on beds of previously printed and dried ceramic powder, as is required for 3D fabrication, coffee staining was observed. This suggests that the morphology of a surface may influence the mechanism of coffee stain formation. Thus the objective of this work is to further investigate the coffee staining process after inkjet printing onto porous surfaces, when solvent removal occurs by capillary driven transport into the substrate in parallel with drying through solvent evaporation.

\section{EXPERIMENTAL METHOD}

A concentrated suspension of ceramic particles was chosen as a model fluid for this study. This is because an important application for printing on porous substrates is the 3dimensional printing of ceramics. ${ }^{7}$ However, the results presented here are believed to be appropriate for the printing of other particle suspensions on porous substrates. The ink used consisted of an aqueous solution of $10 \mathrm{wt} \%$ poly(ethylene glycol) - PEG (BDH, Poole, UK) containing $\mathrm{ZrO}_{2}$ nanoparticles with mean diameter $\approx 140 \mathrm{~nm}(99.99 \%$ Tosoh-Zirconia, TZ3YS-E, Tosoh Corp., Yamagushi, Japan) stabilized in suspension with commercial 
surfactants. Full details of the composition, preparation and rheological characterization of the $\mathrm{ZrO}_{2}$ inks are given elsewhere. ${ }^{22}$

The coffee staining behavior of inkjet printed drops was investigated on a solid metal sheet (Ti-6Al-4V alloy, Goodfellow, Cambridge, UK), photographic quality printing paper (FujiFilm Corporation, Tokyo, Japan), filter paper (Whatman International Ltd., Maidstone, UK) and dried powder beds of the following ceramics: Tosoh-Zirconia, TZ-3YS-E, mean particle size $140 \mathrm{~nm}$, alumina powders A35 and A300 (PI-KEM Ltd., Wem, UK) with mean particle size $35 \mathrm{~nm}$, and $300 \mathrm{~nm}$ respectively . Finally a substrate made from Plaster of Paris (hydrated calcium sulphate) was investigated, because such materials are commonly used to remove water from ceramic suspensions through their use in "ceramic slip casting", where particle suspensions are poured into highly porous moulds, which remove the suspending liquid through capillary action while retaining the suspended powder by filtration. Receding contact angles of the drops were measured by applying $\approx 5 \mu$ volume drops of the fluid on the appropriate substrate and recording the time evolution of the contact angle of the resulting sessile drop using image analysis software (FTA 200, Camtel, Royston, U.K.). For this size of drop the Bond number $<0.1$ and thus the shape of the sessile drop was assumed to be a spherical cap. All experiments were carried out under laboratory conditions at a temperature of approximately $20{ }^{\circ} \mathrm{C}$ under ambient humidity. A number of experiments using alumina powder beds were carried out with the substrate heated to $60{ }^{\circ} \mathrm{C}$.

Zirconia suspensions were printed onto flat surfaces using an in house designed and built laboratory scale inkjet printing platform (Micromech Systems, Braintree, UK). This uses 
piezoelectric inkjet printheads of diameter $60 \mu \mathrm{m}$ (MJ-ATP-01, Microfab Technologies, Plano, TX, USA) with drive electronics (JetDrive III, Microfab) interfaced to a PC and controlled in a LabVIEW ${ }^{\mathrm{TM}}$ environment (National Instruments, Austin, TX, USA). Inkjet printing parameters were a single actuating pulse of $80 \mathrm{~V}$ with $3 \mu$ s rise time, $10 \mu$ s dwell time and $3 \mu$ s fall time at a frequency of $200 \mathrm{~Hz}$. The choice of appropriate printing conditions for any given fluid is governed by the acoustic properties of the printhead/fluid combination used. The principles behind the appropriate choice of conditions have been discussed in detail elsewhere. ${ }^{26}$ Both isolated drops and lines produced from trains of overlapping drops have been printed to study coffee staining during film drying.

The dimensions of printed structures were measured using optical microscopy and a white light interferometric system (MicroXAM Surface Mapping Microscope: Phase Shift Technology Inc., Tucson, AZ, USA); images of the surface profile of drops were created by "stitching together" a series of profilometer line scans from the interferometer.

\section{RESULTS AND DISCUSSION}

Figure 1 shows the shape of dried sessile drops and printed lines on a solid metal (fig. 1a and 1c) and porous powder bed (fig. $1 \mathrm{~b}$ and $1 \mathrm{~d}$ ) substrates using the $\mathrm{ZrO}_{2}$ ink. On the solid surface, the deposit is rounded with a single central peak, while on the porous powder substrate a significant ring deposit, or coffee stain, is visible on the isolated drops and two parallel ridges are located toward the edges of the printed lines. Figure 2 shows tracks printed with 5 printing passes on two paper substrates; in both cases there is a raised edge to the 
printed line indicating the presence of coffee staining. In an earlier report on the ink used in this study, it was observed that coffee stains were also suppressed on solid glass and epoxy resin surfaces. ${ }^{22}$ Thus, with this ink, coffee staining is suppressed on three different solid substrates and is observed on three porous surfaces; hence it is reasonable to assume that the porosity of the substrate strongly influences coffee stain formation.

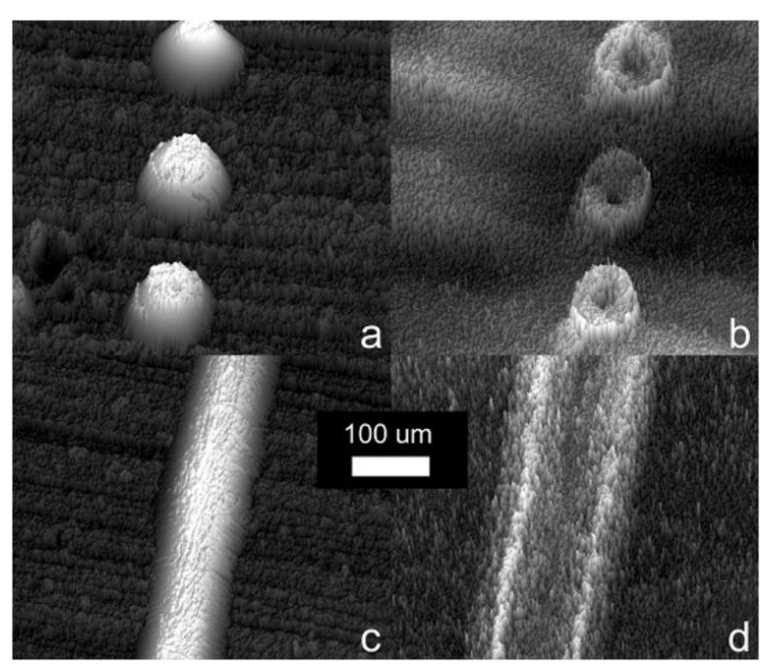

Figure 1 Interferometer images from inkjet printed features using the $\mathrm{ZrO}_{2}$ ink; a) uniform domed drops overprinted after drying on a Ti substrate, b) drops printed on $\mathrm{ZrO}_{2}$ powder bed showing coffee staining, c) line printed on Ti showing uniform profile, d) line printed a $\mathrm{ZrO}_{2}$ powder bed showing raised edges from coffee staining. All features were printed using 10 overprinting passes of the printer.

For the purpose of this study a coffee stain is defined as a distinct height maximum away from the centre of a dried deposit, either as a ring for the case of a single drop, or as two symmetrical ridges for a printed line. Because the height of a dried deposit depends on the contact angle and the degree of overlap of drops, the ratio of the height maximum to the local 


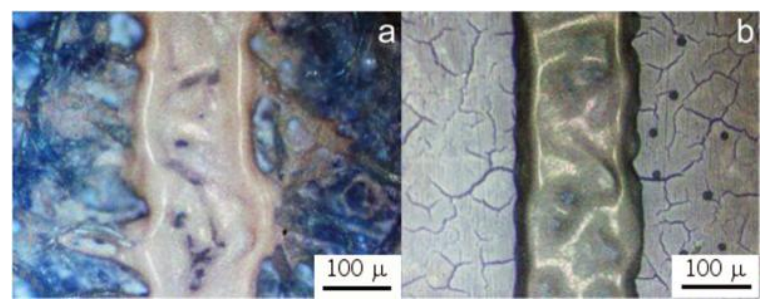

Figure 2 Optical micrographs of inkjet printed $\mathrm{ZrO}_{2}$ lines on: a) filter paper and b) photo quality paper substrates using. A brighter region indicates a raised profile at the edges of the lines on both substrates.

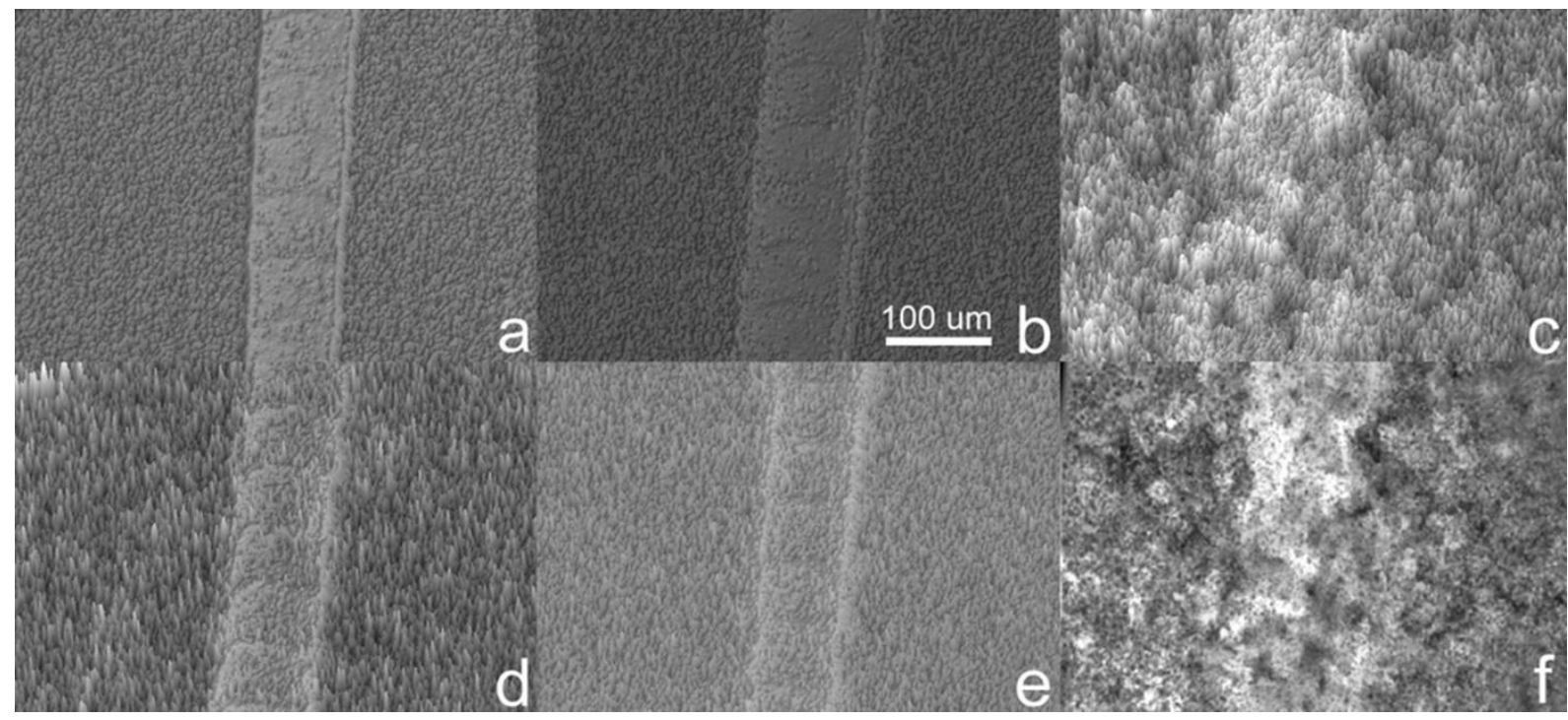

Figure 3 Interferometer images of inkjet printed lines (10 passes) on porous surfaces using the $\mathrm{ZrO}_{2}$ ink on alumina powder beds and Plaster of Paris at two temperatures: a) A35 at $20{ }^{\circ} \mathrm{C}$, b) A300 at $20{ }^{\circ} \mathrm{C}$, c) Plaster of Paris at $20{ }^{\circ} \mathrm{C}$, d) A35 at $60{ }^{\circ} \mathrm{C}$, e) A300 at $60{ }^{\circ} \mathrm{C}$, f) Plaster of Paris at $60{ }^{\circ} \mathrm{C}$.

minimum in the centre of a deposit showing a coffee stain was taken as a suitable metric for the magnitude of a coffee stain. Figure 3 compares the extent of coffee staining seen on 
porous substrates at room temperature and at $60{ }^{\circ} \mathrm{C}$, using powder beds of alumina with mean particle size $35 \mathrm{~nm}$ and $300 \mathrm{~nm}$ and also on a substrate made from Plaster of Paris (hydrated calcium sulphate). Coffee staining is evident on the two powder bed substrates at both deposition temperatures but there is no visible deposit on the plaster substrate. In figure 4 the magnitude of the coffee stains observed on the zirconia and the two alumina powder beds are compared. The larger particle size alumina powder bed shows a greater edge/centre height ratio than the finer alumina powder bed at both temperatures. The coffee staining on the zirconia powder bed was only measured at room temperature but showed a height ratio intermediate to the two alumina powder beds at this temperature.

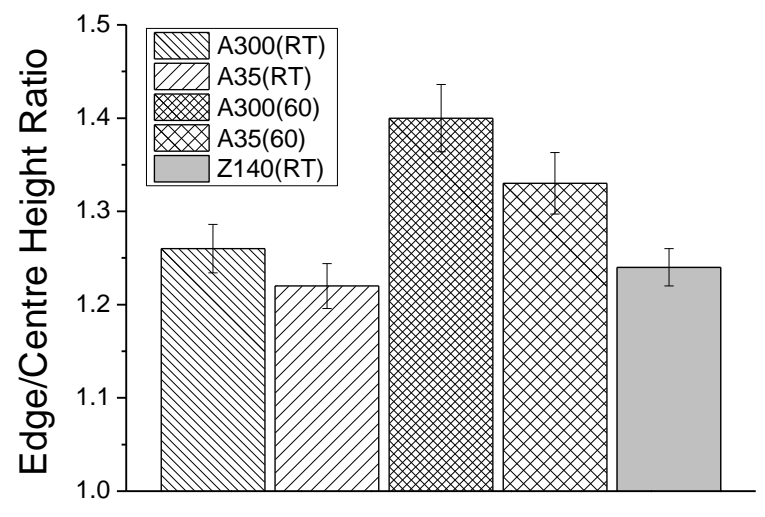

Figure 4 Mean height ratio between the centre and the raised edges of each printed track on the alumina powder beds of two particle sizes (A300 and A35) at room and elevated temperatures and the zirconia powder bed (Z140) at room temperature only. Mean value calculated from 10 printed lines, error bars show standard deviation. 
If coffee staining on porous subjects is driven by solvent removal through capillary draining as well as through solvent evaporation, this can be represented in the simple axisymmetric flow model proposed by Deegan et al. (equation 1$)^{1}$ by including an additional contribution from the liquid draining into the capillary bed with:

$$
\rho \frac{d h}{d t}=-\rho \frac{1}{r} \frac{\partial}{\partial r}(r h v)-J_{1}(r, t)\left(1+\left(\frac{\partial h}{\partial r}\right)^{2}\right)^{1 / 2}-J_{2}(r, t)
$$

where $J_{1}$ and $J_{2}$ are the evaporation and draining fluxes respectively. As can be seen from equation 3 , both these fluxes influence the magnitude of the radial velocity required to maintain a pinned contact line in the same manner, with an increase in either contribution leading to an increased compensating radial flow. There are significant differences in how these two fluxes influence other aspects of fluid behavior and the potential generation of countervailing Marangoni flows. The evaporation flux, $J_{l}$, associated with the drying of solvent mixtures is more complex than equations 1 or 3 imply. Each component of the fluid has its own partial pressure and hence evaporation rate. Thus, during evaporation driven drying, the composition of the fluid may change significantly with time and location across a drying deposit. The resulting local changes in fluid properties may induce Marangoni flows that oppose the radial flow inducing a coffee stain. The draining flux, $J_{2}$, however, is a bulk fluid flow driven by capillary pressure. This will drain all components of the fluid uniformly and is not expected to influence the composition of the drop. In which case, although coffee staining is driven by the sum of the fluxes $J_{1}$ and $J_{2}$, Marangoni flows will only occur if there is a significant evaporation flux, $J_{l}$, enabling local variation in the surface composition of the 
drop. Thus, it is proposed that the coffee stain behavior of drying deposits on a porous surface will be controlled by the relative magnitudes of the fluxes $J_{1}$ and $J_{2}$. We note that we have neglected any interaction between the two fluxes that may occur because the evaporation flux will lead to changes in drop composition with radial position.

In order to consider the likelihood of coffee staining driven by a mechanism of solvent draining, it is necessary to develop models of drop evaporation and draining to allow comparison of the magnitude of the fluxes $J_{1}$ and $J_{2}$. As discussed earlier, the radial variation of the evaporation flux, $J_{l}$, is a topic of discussion in the literature. In contrast the more limited work on the draining of drops into a porous substrate has considered a uniform draining flux, $J_{2}$, across the contact area. ${ }^{10,11,27,28}$ Although, it should be noted that a number of the arguments used to predict a radial variation in $J_{l}$, e.g. presence of a surrounding dry surface or the height of the drop tending to zero at the contact line, have parallels in the case of draining a small isolated drop. However, to simplify our analysis of the relative magnitudes of $J_{1}$ and $J_{2}$, any radial variation in the fluxes is ignored. In this study our observations are made after complete drying of the printed drops, hence expressions are presented for the characteristic times to remove a single component drop by either evaporation or draining and it is assumed that the mean flux can be characterized by its inverse. Although this is a gross simplification of our fluid, which is both a particle suspension and a solution, we believe that this approach will still give considerable insight to assist in the interpretation of the experimental results. 
The evaporation process during the drying of small sessile drops with pinned contact radius has been recently investigated by Butt; ${ }^{29}$ they found that for both pinned (constant contact radius) and unpinned (constant receding contact angle) the time to complete evaporation scaled as $V_{0}^{2 / 3}$, where $V_{0}$ is the drop volume. For the case of a pinned drop the characteristic evaporation time, $\square_{1}$ is given by

$$
\tau_{1}=V_{0} \frac{\rho R T}{2 \pi a D M \Delta P}\left(\frac{f(\theta / \beta)}{\sin (\theta / \beta)}\right)
$$

where $\Delta P, \rho, D$ and $M$ are the difference in vapor pressure above the drop and in the far field, the density of the fluid, the diffusivity of the fluid molecules in air and the molecular weight of the evaporating fluid species respectively; $a$ is the contact line radius $\theta$, is the contact angle of the sessile drop and $\beta$ is a constant of about 1.6. It was found the $\theta \tau$ erm, in parentheses in equation 4 , is only a weak function of $\theta$, varying from 0.65 at $\theta=0^{\circ}$ to 1 at $\theta=90^{\circ}$. Denesuk used the Washburn equation to model the draining of isolated drops into porous media. ${ }^{27,28}$ The time, $\tau_{2}$, to fully drain a liquid drop of volume, $V_{0}$, and pinned spread radius, $a$, is given by

$$
\tau_{2}=\left(\frac{V_{0}}{a^{2} \kappa}\right)^{2}
$$

with $\kappa=\pi A_{p}\left(\frac{\gamma_{L V} r_{p} \cos \theta^{*}}{2 \eta}\right)^{1 / 2}$ 
where $A_{p}$ is the area density of pores of mean radius $r_{p}$; and $\gamma_{L V}$ and $\eta$ are the fluid surface energy and viscosity respectively. In previous work we derived a value of $A p=0.126$ to define the pore area density of a powder bed and this value is used here. ${ }^{11}$ Observations on the absorption of liquid drops into a range of porous media suggest that the pinned contact line approximation is appropriate for the penetration of liquid drops of volume in the range 5 - $10 \mu \mathrm{l}^{27,28}$ This simple model has also been shown in previous work to be applicable to the penetration of inkjet printed drops into porous media, where the drop volume is $\ll<1 \mathrm{nl}{ }^{10,11}$

Inkjet printers produce drops with volume $\ll<1 \mathrm{nl}$, hence sessile drops that form after spreading to equilibrium can be modelled as spherical caps. ${ }^{6}$ For a spherical cap the drop volume is related to the contact radius and contact angle with

$$
V_{0}=\frac{\pi a^{3}}{3 \sin ^{3} \theta}\left(2-3 \cos \theta+\cos ^{3} \theta\right)
$$

Substituting into the relation between contact area and drop volume into equations 4 and 5 gives the following relations for $\tau_{1}$ and $\tau_{2}$

$$
\begin{aligned}
& \tau_{1}=V_{0}^{2 / 3}\left\{\frac{\pi}{3}\left(2-3 \cos \theta+\cos ^{3} \theta\right)\right\}^{1 / 3}\left(\frac{f(\theta / \beta)}{\sin (\theta / \beta)}\right)\left[\frac{\rho R T}{2 \pi D M \Delta P}\right] \\
& \tau_{2}=V_{0}^{2 / 3}\left\{\frac{\pi}{3}\left(2-3 \cos \theta+\cos ^{3} \theta\right)\right\}^{2 / 3} \frac{1}{\cos \theta^{*}}\left[\frac{2 \eta}{\pi^{2} A_{p}^{2} \gamma_{L V} r_{p}}\right]
\end{aligned}
$$


There are considerable similarities between the two equations that define the characteristic time scale for drop evaporation and drainage. Each contains a transport term (within square brackets), a geometry term that can be defined in terms of the contact angle of the drop, and an identical function of initial drop volume. Thus, for the case of small drops and a spherical cap approximation, there is no transition from one mechanism of drop removal to another as a function of drop volume. It is clear that the relative magnitude of the two time scales will be dominated by the transport terms, although there will be a range over which geometry (contact angle) is important.

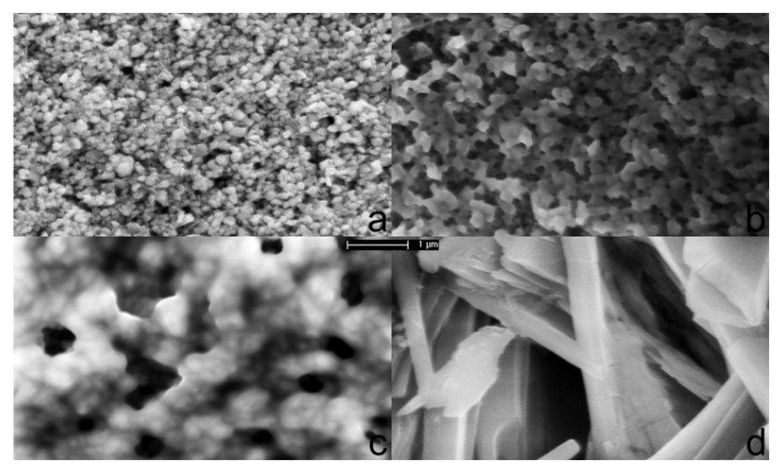

Figure 5 Scanning electron microscopy images of the surface of the porous substrates used in the experiments: a) $35 \mathrm{~nm} \mathrm{Al} 2 \mathrm{O} 3$ powder bed, b) $140 \mathrm{~nm} \mathrm{ZrO} 2$ powder bed produced by inkjet printing, c) $300 \mathrm{~nm} \mathrm{Al} 2 \mathrm{O} 3$ powder bed, d) Plaster of Paris. In substrates a - c the porosity is approximately the same size as the powder particles, while with substrate $d$ the plaster crystals are elongated and mean pore diameter is $>1 \mu \mathrm{m}$.

We can assume that in our experiments the transport conditions for drop evaporation remain constant, independent of powder bed composition, while the transport of fluid into the powder beds is controlled by the pore size. The parameters that vary between each of the 
powder beds used in these experiments will be the pore radius, which is controlled by the particle size and packing density, and the contact angles $\theta$ and $\theta^{*}$. Figure 5 shows scanning electron microscope (SEM) images of the surfaces of the powder beds used in this study; the visible pore diameter appears to be similar to the mean particle diameter of the powders. This is consistent with previous work studying the behavior of inkjet printed drops on powder beds, in which the pore radius was taken to be adequately described by a simple linear function of the particle size. ${ }^{11}$ In figure $5 \mathrm{~d}$ we can clearly see that the porosity of the Plaster of Paris surface is of considerably larger size than the alumina or zirconia powder beds.

It is difficult to determine the appropriate contact angle to use with the Washburn equation, $\theta^{*}$, when working with powder beds. However, it is possible to measure the contact angle, $\theta$, on different powder surfaces using simple sessile drop experiments. Figure 6 shows the evolution of the contact angle with time for $\approx 5 \mu$ drops placed on the porous surfaces. In all cases the contact angle decreases rapidly with time because the pinned contact line prevents the development of a stable receding contact angle, consistent with the observations of Hapgood. ${ }^{30}$ With the Plaster of Paris substrate, the liquid is rapidly drained and no liquid remains on the surface after about 10 minutes. Using Cassie's law we can use this data to estimate the effective contact angle, $\theta^{*}$, used in the Washburn equation and our equation 4 , assuming an effective surface area fraction of fluid filled pores. This approximation has been used successfully in previous studies of inkjet printed drops on powder beds, ${ }^{10,11}$ with

$$
\cos \theta^{*}=\frac{\cos \theta-A_{p}}{1-A_{p}}
$$


where, $\theta$, is the contact angle measured on the powder bed and $A_{p}$ is the fraction of exposed surface porosity (taken as 0.126 in this case, consistent with earlier studies ${ }^{10,11}$ ).

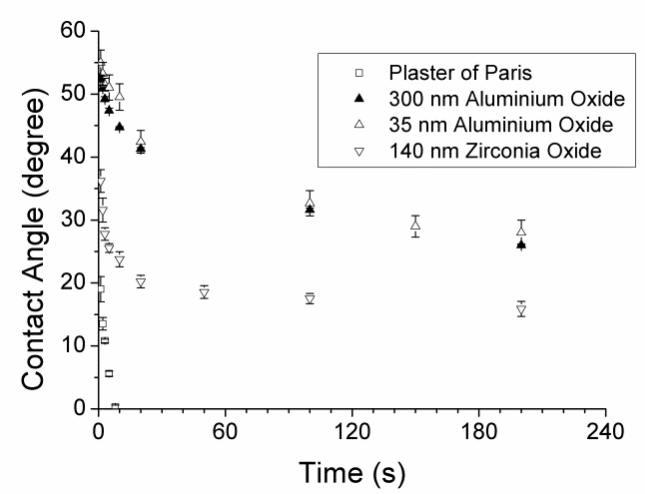

Figure 6 Contact angle measured from $5 \mu$ drops of the $\mathrm{ZrO}_{2}$ suspension on a range of porous substrates at room temperature.

In order to explore whether our observations of the behavior of the ceramic ink are consistent with our proposed mechanism, we explore how the characteristic time for evaporation and draining (equations 7 and 8) varies with porosity radius and contact angle. The fluid physical properties used in these calculations are listed in Table 1 for the zirconia ink and also, for comparison, pure water at $298 \mathrm{~K}$. We use the ratio $\tau_{1} / \tau_{2}$ to characterize the positions of the $\mathrm{Al}_{2} \mathrm{O}_{3}$ and $\mathrm{ZrO}_{2}$ powder beds are given, with a circle indicating the use of $\theta_{0}$ and a cross $\theta_{20}$. The dashed line at the top of the plots indicates where $\theta^{*}=90^{\circ}$ and there is thus no driving force for capillary draining. However, for draining to induce coffee staining it is not clear whether its flux is simply required to be greater than that for evaporation or whether a lower but significant flux will suffice, hence we plot in figure 7 contours for $\tau_{1} / \tau_{2}=0.1,0.3$, 1,3 and 10 as a function of contact angle on the powder bed and mean pore radius. 


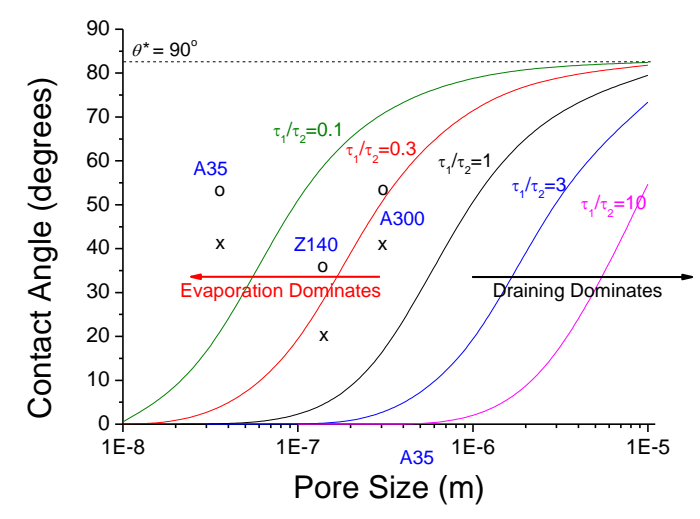

b)

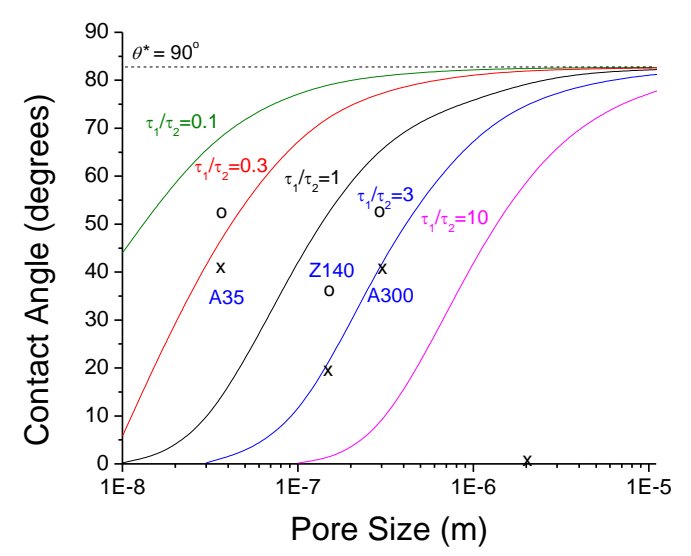

Figure 7 The influence of pore size and contact angle on the ratio of characteristic time for evaporation and drainage for a) the $\mathrm{ZrO}_{2}$ ink, b) water drops, both at $293 \mathrm{~K}$. On both plots the fluid removal mechanism from a sessile drop. If $\tau_{1} / \tau_{2}>1$ the draining flux is expected to be greater than evaporation, and conversely evaporation is expected to dominate if $\tau_{1} / \tau_{2}<1$. 
Table 1 Fluid physical properties used in equations 7 and 8.

\begin{tabular}{|c|c|c|c|c|c|}
\hline & $\begin{array}{l}\text { Density } \\
\left(\mathrm{kgm}^{-3}\right)\end{array}$ & $\begin{array}{l}\text { Viscosity } \\
\text { (mPa.s) }\end{array}$ & $\begin{array}{l}\text { Surface } \\
\text { Tension } \\
\left(\mathrm{mJ} . \mathrm{m}^{-2}\right)\end{array}$ & $\begin{array}{l}\text { Diffusion } \\
\text { Coefficient in } \\
\text { Air }\left(\mathbf{m}^{2} \mathbf{s}^{-1}\right)\end{array}$ & $\begin{array}{l}\text { Eqm. Vapor } \\
\text { Pressure } \\
\text { (kPa) }\end{array}$ \\
\hline $\mathrm{ZrO}_{2} \operatorname{Ink}^{17}$ & 1012 & 4.51 & 48 & $2.4 \times 10^{-5}$ & $2.08^{*}$ \\
\hline $\mathbf{H}_{2} \mathbf{O}$ & 997 & 0.85 & 72 & $2.4 \times 10^{-5}$ & 2.31 \\
\hline
\end{tabular}

*Assuming ideal solution

On inspecting figure 7 it is clear that for the case of liquid drops of the ink or water on powder beds with a mean pore radius $>1 \mu \mathrm{m}$ fluid drainage into the bed is expected to occur at a faster rate than fluid loss through evaporation, if $\theta^{*}<90^{\circ}$. From figure 6 , the contact angle of a sessile drop on the powder bed decreases rapidly with time. However, what is not so evident is the value of the contact angle appropriate for use in equations 6 and 7. That value is the assumed "equilibrium angle" once sufficient fluid has been adsorbed to provide a composite surface as modelled using Cassie's law. It is unclear how much of the rapid initial decrease in contact angle is caused by volume loss through draining and how much by adjustment of the energy balance. To allow for this uncertainty we consider two values for the contact angle on the powder bed at time $=0$ giving $\theta_{0}=53^{\circ}$ on $\mathrm{Al}_{2} \mathrm{O}_{3}, \theta_{0}=36^{\circ}$ on $\mathrm{ZrO}_{2}$ and at time $=20 \mathrm{~s}$ giving $\theta_{20}=41^{\circ}$ on $\mathrm{Al}_{2} \mathrm{O}_{3}, \theta_{20}=20^{\circ}$ on $\mathrm{ZrO}_{2}$. Figure $7 \mathrm{a}$ indicates the relative draining and evaporation fluxes, from the contours we can see that both the coarse $\mathrm{Al}_{2} \mathrm{O}_{3}$ and $\mathrm{ZrO}_{2}$ powder beds show similar behavior with the finer $\mathrm{Al}_{2} \mathrm{O}_{3}$ powder bed predicted to show a greater influence of evaporation. This is consistent, within experimental uncertainty) with the relative amounts of coffee staining seen in figure 4 . The location of the 
transition behavior in figure $7 \mathrm{a}$ for the zirconia ink appears to be at a significantly larger substrate pore size than we find experimentally. The transition predicted with water in figure $7 \mathrm{~b}$ appears closer to our observations. However, considering the approximate nature of our model and that the zirconia ink is a complex multicomponent mixture, it is the relative behavior of the powder beds that is important.

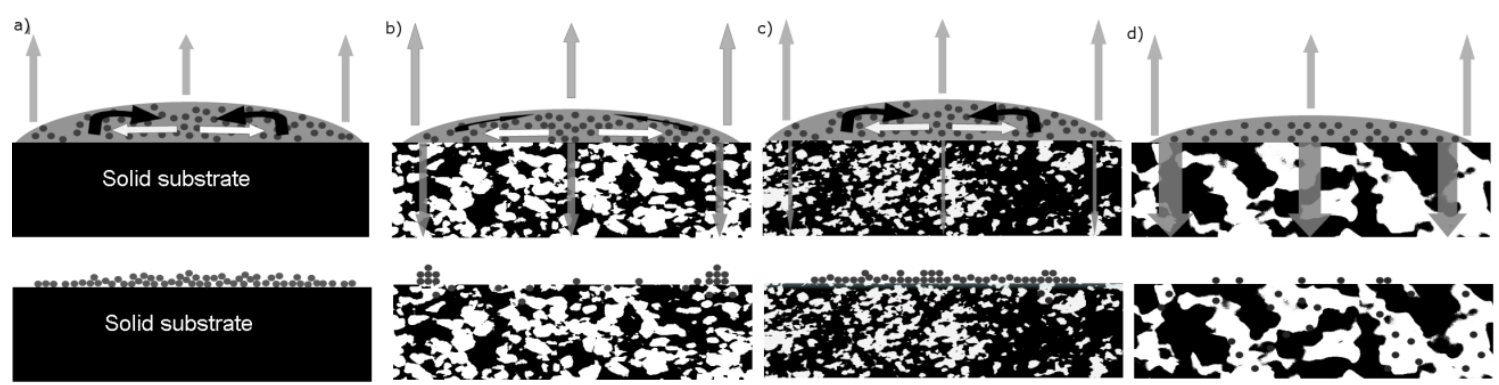

Figure 8 Schematic representation of relative fluxes from draining and evaporation (grey arrows), internal radial flow (white arrows) and Marangoni flow (black arrows) leading to different coffee stain behavior: a) $\mathrm{ZrO}_{2}$ ink on a solid surface - PEG addition leads to differential evaporation and Marangoni flow opposes radial flow eliminating any coffee stain; b) on a porous substrate radial flow is enhanced by the draining of fluid into the pores, reduced evaporation flux leads to reduced Marangoni flow and coffee staining occurs; c) below a critical pore size the draining rate is too slow and evaporation drying dominates as with a solid substrate; d) if the porosity is large, fluid drains into substrate without filtration, eliminating coffee stains and surface deposition.

It is proposed that the deposition behavior seen on porous substrates depends on the relative magnitude of the two solvent fluxes, $J_{1}$ and $J_{2}$ used in equation 1 . On a solid surface $J_{2}=0$ and the drop dries through evaporation, in this case the $\mathrm{ZrO}_{2}$ ink has been optimized to 
suppress coffee staining through modification of the evaporation flux. ${ }^{18}$ and a countervailing Marangoni flow suppresses segregation during drying (figure 8a). On the powder beds formed from alumina and $\mathrm{ZrO}_{2}$ particles the porosity is such that solvent drainage occurs significantly more rapidly than solvent evaporation (figure 8b). The draining flux does not lead to changes in solvent composition and thus any driving force for Marangoni convection can only be generated by the smaller evaporation flux, this reduction in Marangoni flow results in the formation of a coffee stain. However the draining flux is a function of particle size and if the powder bed is made from smaller diameter powder, the draining flux is reduced (equation 2). Below some critical particle size (figure 8c) the evaporation flux is sufficiently greater than the draining flux, solvent loss now behaves in a similar manner to the solid surface and coffee staining is suppressed by Marangoni flows. On the plaster surface, the porosity is significantly larger than the mean diameter of the particles in suspension, hence the suspension drains into the porous surface without filtration, leaving no surface deposit (figure 8d).

The extent of coffee staining seen in our experiments scales inversely with powder particle size for the $\mathrm{Al}_{2} \mathrm{O}_{3}$ powder beds at both temperatures, a trend that is consistent with the predicted solute draining times obtained from Washburn's equation and Butt's evaporation model. The coffee stain metric from experiments on the $\mathrm{ZrO}_{2}$ powder bed (room temperature only) is, from figure 4 , intermediate to that of the larger and smaller $\mathrm{Al}_{2} \mathrm{O}_{3}$ powders. However, from the location of the powder bed properties on the contour plots of figure 7 , our model suggests that the behavior of the $140 \mathrm{~nm} \mathrm{ZrO}_{2}$ and the $300 \mathrm{~nm} \mathrm{Al}_{2} \mathrm{O}_{3}$ powder should be equivalent. We recognize that the models presented in equations 7 and 8 and presented in 
figure 7 are gross oversimplifications in that they use models for single component fluids and ignore the presence of solute and particles. Thus an exact match with our experimental data would be unlikely. However, the fact that the calculations indicate a transition from evaporation to draining dominated fluid removal at a pore radius close to those used in our study indicates that the proposed mechanism is physically reasonable.

From figure 4 it can be seen that increasing the substrate temperature increases the extent of coffee staining for both alumina powder beds. This is consistent with Ozawa's mechanism, ${ }^{14}$ where an increase in substrate temperature leads to a rapid rise in temperature at the drop edges because of the reduced height of the liquid column. This local increase in temperature will lead to an increase in the evaporation flux and also, because of the reduction in viscosity with increasing temperature, an increase in the draining flux close to the contact line. An increase in both fluxes $J_{1}$ and $J_{2}$ close to the contact line will lead to an increased driving force for coffee staining following Deegan's analysis. ${ }^{1,4}$

Finally note that the implicit assumption, that draining driven coffee staining also requires the surface porosity to filter the nanoparticles, may not be a necessary requirement. Wang et al reported on the adsorption of protein solutions printed as continuous lines on a porous membrane, ${ }^{31}$ finding that protein segregated towards the edge of the lines after drying. However, their analysis found a number of distinct differences between their observed segregation and that expected from conventional coffee staining. In particular they found that segregation occurred during the early stages of drop drying and was associated with the draining phase of liquid penetration into the porous membrane rather than during the removal 
of solvent by evaporation. Thus the proposed enhanced concentration of solvent during drainage leads to selective deposition without the need for particle filtration.

\section{CONCLUSIONS}

These results indicate that in the case of the model $\mathrm{ZrO}_{2}$ ink, solvent removal through draining into a porous bed leads to ring deposits, or coffee stains, at the edges of drying drops of particulate suspensions. Such coffee stains are not seen when the same ink is used on solid surfaces. Coffee stains are formed through a radial flow needed to maintain the pinned contact line at the edge of a drying drop or printed line; this leads to an increase in concentration of particulate material, which is filtered from the solvent by the fine size of the porous substrate. This mechanism is identical to that proposed by Deegan ${ }^{1}$ for the formation of coffee stains during drying by evaporation. Simple models for the relative importance of the evaporation and draining fluxes (developed in terms of characteristic times) show that a draining flux, of comparable magnitude to the evaporation flux occurs with alumina and zirconia powder beds with particle sizes around $100 \mathrm{~nm}$.

However, there are significant differences between the mechanisms of coffee staining driven by evaporation and capillary draining. The most important of these is that capillary draining removes the individual components of the liquid from the drop in proportion to its composition, while evaporation will remove the individual components of the liquid at a rate dependant on their vapor pressure. Hence, whereas drying of a sessile drop through 
evaporation can lead to Marangoni flows, which may oppose the coffee staining radial flow, drying through capillary drainage cannot generate such flows.

The relative magnitude of the evaporation and draining fluxes strongly influences the presence of coffee stains on porous surfaces. Simple models of drying and draining show that the relative evaporation and drying fluxes are expected to be independent of drop size and thus we expect the coffee staining phenomenon to be also independent of drop size. As the pore size of the substrate increases, the draining flux increases and a greater tendency for coffee staining occurs, this behavior is modified by the contact angle of the drop. However, there is a maximum substrate pore size, above which no coffee staining is seen because the pore size become too large to efficiently filter the particles from the fluid at the substrate surface. We note that because capillary draining does not change the composition of the liquid phase, many of the strategies employed to minimize coffee staining through evaporation, by the generation of Marangoni flow, will not be applicable when the phenomenon is driven through capillary draining.

Further work is clearly needed to develop more appropriate models that incorporate evaporation, draining and Marangoni flows. A further development of the model to include the influence of a multicomponent solution (or at least a model for a binary solution) would also improve our understanding of the process. 


\section{REFERENCES}

1. Deegan, R. D.; Bakajin, O.; Dupont, T. F.; Huber, G.; Nagel, S. R.; Witten, T. A., Nature 1997, 389 (6653), 827-829.

2. Joanny, J. F.; de Gennes, P. G., J. Chem. Phys. 1984, 81, 552-563.

3. Nadkarni, G. D.; Garoff S., Europhys. Lett. 1992 20, 523-528.

4. Deegan, R. D.; Bakajin, O.; Dupont, T. F.; Huber, G.; Nagel, S. R.; Witten, T. A., Physical Review E 2000, 62, 756-765.

5. Davis, S. H., J. Fluid Mech. 1980, 98, 225-242.

6. Stringer, J.; Derby, B., Langmuir 2010, 26, 10365-72

7. Derby, B., J. Europ. Ceram. Soc. 2011, 31, 2543-2550.

8. Wood, L. K.; Hrehorova, E.; Joyce, T. W.; Fleming, P. D.; Joyce, M.; Pekarovicova, A.; Bliznyuk, V., Paper substrates and inks for printed electronics, in Pira Ink on Paper Symposium, Atlanta, GA, September 2005,

9. Gimpel, S.; Möhring, U.; Müller, H.; Neudeck, A.; Scheibner, W., J. Industrial Textiles, 2004, 33, 179-189.

10. Holman, R. K.; Cima, M. J.; Uhland, S. A.; Sachs, E., J. Coll. Interface Sci. 2002, 249, 432-440.

11. Wang, T. M.; Patel, R.; Derby, B., Soft Matter 2008, 4, 2513-2518.

12. Fischer, B. J., Langmuir 2002, 18, 60-67.

13. Hu, H.; Larson, R. G., Langmuir 2005, 21, 3972-3980.

14. Ozawa, K; Nishitani, E; Doi, M., Jap. J. Appl. Phys. 2005, 44, 4229-4234.

15. Hu, H.; Larson, R. G., J. Phys. Chem. B 2006, 110, 7090-7094. 
16. Poulard, C.; Guena, G.; Cazabat, A. M., J. Phys.: Condens. Matter, 2005, 17, 5421354227.

17. Soltman, D.; Subramanian, V., Langmuir, 2008, 24, 2224-2231.

18. Dou, R.; Derby, B., J. Amer. Ceram. Soc. 2011, 94, 3787-3792.

19. Wang, T; Roberts, M. A.; Kinloch, I. A.; Derby, B., submitted to J. Phys. D, Appl. Phys..

20. Yunker, P. J.; Still, T.; Lohr, M. A.; Yody, A. G., Nature, 2011, 476, 308-311.

21. Shen, X.; Ho, C. M.; Wong, T.S.; J. Phys. Chem. B 2010, 114, 5269-5274.

22. Derby, B., Ann. Rev. of Mater. Res., 2010, 40, 395-414.

23. de Gans, B. J.; Schubert, U. S., Langmuir 2004, 20, 7789-7793.

24. Park, J.; Moon, J., Langmuir 2006, 22, 3506-3513;

25. Zhang, Y.; Yang, S.; Chen, L.; Evans, J. R. G., Langmuir 2008, 24, 3752-3758.

26. Reis, N.; Ainsley, C.; Derby, B., J. Appl. Phys. 2005, 97, 094903.

27. Denesuk, M.; Smith, G. L.; Zelinski, B. J. J.; Kreidl, N. J.; Uhlmann, D. R., J. Coll. Interface Sci. 1993, 158, 114-120;

28. Denesuk, M.; Zelinski, B. J. J.; Kreidl, N. J.; Uhlmann, D. R., J. Coll. Interface Sci. 1994, 168 (1), 142-151.

29. Schönfeld, F.; Graf, K-H.; Hardt, S.; Butt, H-J., Int. J. Heat Mass Transfer, 2008, 51, 3696-3699.

30. Hapgood, K. P.; Lister, J. D.; Biggs, S. R.; Howes, T., J. Coll. Interface Sci. 2002, 252, 353-366.

31. Wang, J.; Thom, V.; Hollas, M.; Johannsmann, D., J. Membrane Sci. 2008, 318 (1-2), 280-287. 